\title{
Televisión, cine y publicidad, fuentes de conocimiento del pasado y del presente
}

\author{
Pedro Molina Rodríguez-Navas \\ Universitat Autònoma de Barcelona \\ pedro.molina@uab.cat \\ Núria Simelio Solà \\ Universitat Autònoma de Barcelona \\ nuria.simelio.sola@uab.cat \\ Jordi IBARZ GELABERT \\ Universitat de Barcelona. \\ jordi.ibarz@ub.edu
}

\section{Resumen}

Los medios de comunicación son una fuente de estudio del pasado y del presente que permiten mostrar la articulación de los cambios sociales colectivos con las transformaciones individuales.

En este artículo presentamos un método de análisis que hemos experimentado con productos cinematográficos, televisivos y de publicidad. El procedimiento revela la realidad de la pluralidad social al mostrar a todo tipo de protagonistas en los escenarios de la vida pública y privada. La sistematización que proponemos supera los análisis basados en percepciones generales sobre contenidos.

Palabras clave: Publicidad; ficción televisiva; cine; ciudadanía; educación mediática.

\section{Television, Cinema and Advertising, as a Source for Past and Present Knowledge}

\begin{abstract}
Mass Media are a source of research for past and present knowledge that permit to show the articulation between social and collective changes with the individual transformations.

In this article, we present a method of analysis that we have experimented with film, television and advertising products. Our procedure permits to reveal the plural social reality due that it shows a lot of different types of individual humans in public and private spaces. Our proposed methodology has exceeded the content analysis based on general perceptions.
\end{abstract}

Key Words: Advertising; TV fiction; movies; citizenship; media literacy

\section{Referencia normalizada:}

Molina Rodríguez Navas, P., Simelio Solà, N. e Ibarz Gelabert, J. (2013) Televisión, cine y publicidad, fuentes de conocimiento del pasado y del presente. Historia y Comunicación Social. Vol. 18. No Especial Diciembre. Págs. 461-471.

Sumario: 1.Introducción. 2. Propuesta metodológica de análisis de productos audiovisuales. 2.1. Objetivos y antecedentes metodológicos. 2.2. Producción y producto. 2.3. Contextualización: otras fuentes. 2.4. La presentación del trabajo final. 3. Valoración de los estudiantes. 4. Conclusiones. 5. Bibliografía. 


\section{Introducción}

El proyecto que presentamos en este artículo surgió en el marco de la docencia de las asignaturas de Historia de la Comunicación, a partir de la necesidad de repensar las metodologías docentes a la vista de los grandes cambios sociales experimentados en las últimas décadas, particularmente evidentes en la comunicación social. Igualmente, para adaptar los programas a los criterios del EEES, era necesario revisar los trabajos prácticos que se estaban encargando a los estudiantes.

Se partió de una propuesta anterior (Moreno, 1999) que contemplaba la necesidad de estudiar la implantación de los medios de comunicación articulando las historias personales con la historia colectiva, utilizando para ello los productos de los medios de comunicación al considerarlos registros de la memoria. Esta utilización requiere procedimientos específicos que presten atención a la representación social que se ofrece, evidenciando como se narran las relaciones entre los acontecimientos sociales y los personales, para dar cuenta de la pluralidad social en cada momento y lugar. Otras explicaciones de las dinámicas sociales muestran tan solo a personas y hechos que actúan preferentemente en instituciones de poder, ocultando aspectos de la realidad social (Moreno, 1986).

Se concretó este planteamiento diseñando un método de análisis de la publicidad en cualquier soporte y de los audiovisuales de ficción, tanto cinematográficos como televisivos, como fuentes de conocimiento del pasado reciente y del presente. El procedimiento fue pensado para que los estudiantes pudieran aplicarlo en trabajos realizados en grupo que posteriormente debían exponer, aspecto al que se concedió mucha importancia, así que el formato de presentación de resultados forzó el desarrollo de competencias en el campo de la comunicación y el periodismo.

Este trabajo, dirigido por la Dra. Amparo Moreno Sardà, contó inicialmente con la financiación del Vicerrectorado de Estudios y de Calidad de la Universidad Autónoma de Barcelona, a través de un Proyecto de Mejora de la Calidad Docente titulado "Guías Didácticas de Historia de la Comunicación para experimentar las posibilidades de Internet2 para la producción de contenidos de forma cooperativa e interactiva: la publicidad y los programas de televisión como fuentes documentales para comprender las transformaciones sociales personales y colectivas".

La alfabetización mediática forma parte de un conjunto de aprendizajes en lectura comprensiva y crítica (Pérez, 2011) y es necesaria para incentivar la participación democrática (Kellner; Share, 2007; Francisco, 2010). Algunos trabajos destacan la necesidad de implantarla para formar en valores, destacando las posibilidades del material cinematográfico (Ambrós, Breu, 2007) o, en menos ocasiones, de los audiovisuales televisivos (Buckingham, 2003; Medrano, 2008). Otros estudios subrayan la capacidad de estos medios como constructores de memoria (Sánchez-Biosca, 2006; Ibáñez, Anania, 2010; Hernández, 2012) o han señalado carencias como la escasa atención prestada a las mujeres directoras de películas por sus negativas consecuencias en las prácticas docentes (Núñez, 2010). 
Se han hecho propuestas de utilización de los audiovisuales (Gómez, 2010), algunas recurriendo concretamente al cine español (Navarrete, 2009; Valero, 2010), en algunos casos como propuestas de aplicación en enseñanza superior (Palao, 2007) y también incidiendo en los estudios de comunicación (Gómez, 2007).

El procedimiento que nosotros proponemos contempla exclusivamente las producciones de ficción no histórica, es decir, aquellas en que producción y trama son coetáneas. Esto obliga a identificar los elementos de la producción de forma ordenada, describiendo primero para interpretar después, en sintonía con las apreciaciones de Marzal (2007).

En relación a la publicidad, se han hecho trabajos sobre su historia en España (Montero, 2010), sobre su función para el conjunto del sistema económico (Colón, 2001) y otros ya han subrayado su utilidad como fuente para la historia (Fowles, 1996; Sopeña, 1994). Destacamos el método de análisis de Arregui (2009) -que utiliza carteles de propaganda política- porque incorpora variables sobre los roles representados por los seres humanos, aspecto sobre cuya relevancia coincidimos.

\section{Propuesta metodológica de análisis de productos audiovisuales}

\subsection{Objetivos y antecedentes metodológicos}

El objetivo de esta metodología parte de considerar que la publicidad y la ficción audiovisual muestran las transformaciones sociales colectivas y las de los diferentes grupos humanos en cada etapa histórica. Los arquetipos mostrados en los productos mediáticos determinan modelos de integración y/o de marginación, ya que la incorporación a la sociedad de consumo, o la movilidad en la escala social, precisa de elementos materiales y también de modelos a seguir.

Los antecedentes de esta metodología surgen del recorrido del grupo investigador que en los años 80 empezó con un análisis de los manuales de historia de educación secundaria (Moreno, 1986) para seguir una década después con las noticias de prensa (Moreno, 1998). Tras estos trabajos iniciales se formuló un método que posteriormente fue aplicado al análisis de la prensa de la transición política en España (Simelio, 2007) y a la representación del trabajo femenino en la prensa escrita (Rovetto, 2010). Posteriormente, la metodología fue adaptada para que los profesionales de la comunicación pudieran analizar sus propias informaciones (Moreno, Rovetto, Buitrago, 2007). Además, se han realizado experiencias educativas e investigaciones considerando los documentos audiovisuales, los relatos orales y los documentos personales de todo tipo como fuentes de la memoria (Molina, 2007).

Finalmente, el diseño del procedimiento de análisis para el estudio de los audiovisuales y de la publicidad contempla cuatro etapas: análisis de la producción, del producto, validación con otras fuentes y conclusiones. 
2.2. Producción y producto

Gráfico 1: análisis de la producción y el producto

\section{Producción}
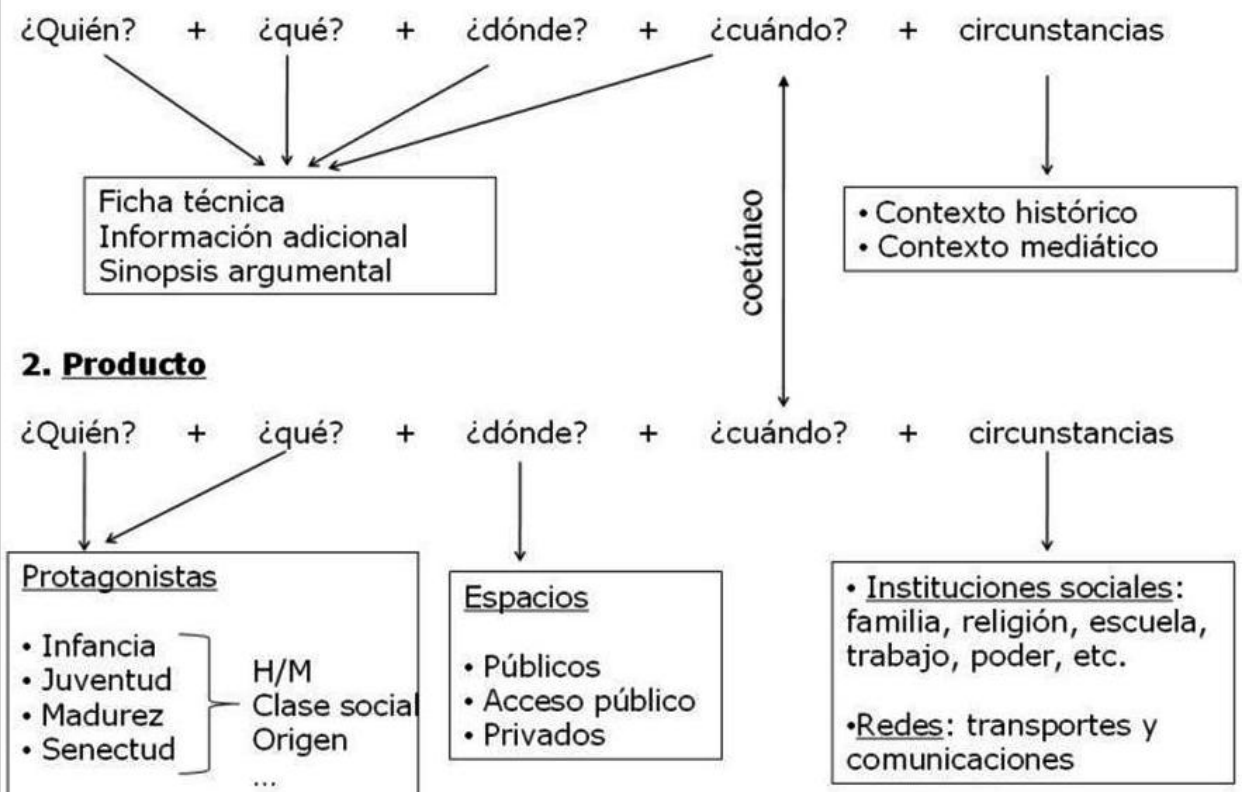

Fuente: elaboración propia

Los estudiantes pueden aplicar el procedimiento entendiendo que la ficción debe ser verosímil y que para ello debe representar los escenarios sociales, las instituciones y las redes de transporte y de comunicación.

Se elaboraron dos guías didácticas, para el análisis de los audiovisuales y para el de la publicidad, con descripciones detalladas del procedimiento y con modelos para la presentación de resultados elaborados a partir de ejemplos.

Como puede verse en el (Gráfico 1) en el análisis de la producción y del producto se abordan aspectos relativos a las historias personales en relación a la historia colectiva: quién hace qué, en qué espacios, cuándo y en qué circunstancias sociales.

Para el análisis de la producción se elabora una ficha técnica que ha de incluir la sinopsis argumental, si se trata de un audiovisual, a la que se suma la descripción del contexto histórico y mediático. El análisis del producto sistematiza los elementos que 
nos interesan: protagonistas, espacios, instituciones sociales y redes de transportes y de comunicaciones.

Si se estudian productos publicitarios se contempla un análisis diacrónico a partir de análisis individualizados (dos o más anuncios de diferentes momentos sobre un mismo tipo de productos, misma marca comercial u organización...) que se contrastan en las conclusiones.

Cuando se trabaja con audiovisuales se analiza uno solo, y es esencial que se trate de una ficción en que realización y relato de ficción sean coetáneos. Se descartan, por tanto, todas las producciones de carácter histórico puesto que lo que interesa es conocer como se han representado a sí mismas las sociedades contemporáneas. Las conclusiones del trabajo deben destacar qué información aporta el material estudiado para la comprensión de las transformaciones sociales.

El análisis del producto es la parte más importante del trabajo. Se han de identificar las características de los personajes, las de los espacios donde desarrollan sus actuaciones y la forma en que aparecen representadas las instituciones sociales y las redes de comunicación. Para el análisis de la publicidad se atiende al texto en un apartado específico, pero para los audiovisuales no resulta necesario ya que los diálogos sirven para caracterizar a los personajes o para identificar aspectos del resto de elementos tratados.

Los estudiantes deben identificar a los personajes, describirlos y organizar su presentación siguiendo las etapas de la vida (infancia, juventud, madurez, senectud) y considerando categorías tales como clase social, diferenciación sexual, colectivo nacional o cultural, profesional, etc. Relacionando estos dos factores (generación-clasificaciones sociales) se observan las condiciones de vida de cada momento para cada colectivo. La descripción de los escenarios públicos y privados amplía la caracterización social, mostrando los espacios en que se dan las diferentes formas de vida de cada momento.

Por último, se abordan las instituciones como formas de organización de la vida social (la familia, las administraciones y poderes públicos, las instituciones religiosas, educativas, culturales, etc.) y las redes de comunicación y de transportes en relación a las necesidades y expectativas de los diversos grupos.

Es condición imprescindible describir todos estos elementos antes de concluir, para que no resulten predominantes ideas preconcebidas, aunque sin olvidar que la ficción precisa de amplificaciones, distorsiones y ocultaciones, por lo que resulta necesario contrastar los resultados del análisis con otras fuentes.

\subsection{Contextualización: otras fuentes}

Para validar la información registrada, distinguiendo elementos útiles de otros que pueden ser distorsiones de la realidad, hay que recurrir a otras fuentes, tales como bibliográficas, documentales, mediáticas o fuentes de la memoria. Esta es una cuestión central para que los estudiantes discriminen elementos fantásticos de otros 
que resulten ser información histórica válida. Privilegiamos en este caso las fuentes orales y el resto de fuentes de origen privado, como fotografías personales, así como la comparación con otros productos de los medios de comunicación (audiovisuales, fotografías, textos periodísticos, etc.).

Aunque también es importante utilizar bibliografía o recursos digitales, muchas veces estas fuentes no dan cuenta de una gran variedad de aspectos de la vida privada de las personas, así que es esencial obtener información de personas que vivieron la época estudiada, mediante sus propios recuerdos o documentos personales. También así se consigue que los estudiantes valoren las vivencias de personas cercanas de forma positiva, como fuentes de información y de conocimiento social.

\subsection{La presentación del trabajo final}

La presentación de los trabajos al resto de estudiantes permite sumar y contrastar análisis, de forma que los resultados parciales se complementan con otros que pueden ser de tipología o intencionalidad muy diversa. La exposición cronológica permite apreciar las transformaciones sociales que se han producido, década a década, hasta la actualidad.

Con esta finalidad, resultan útiles los programas habituales de presentación de diapositivas que permiten incorporar textos, imágenes o hiperenlaces, propiciando una lectura reticular que implica un trabajo próximo a la escritura en los medios digitales actuales.

Igualmente, los enlaces internos entre las diferentes diapositivas muestran las relaciones entre las diferentes partes del trabajo, así como los enlaces externos pueden llevar a otros productos mediáticos con que comparar los analizados.

\section{Valoración de los estudiantes}

Al finalizar el primer curso de experimentación del método (2007-2008) se elaboró una encuesta para conocer la opinión de los estudiantes que habían realizado sus trabajos de curso aplicando el método de análisis propuesto. Estos estudiantes cursaban tres titulaciones: periodismo, comunicación audiovisual y publicidad y relaciones públicas. Las encuestas constaban de una veintena de preguntas referidas a la valoración de las guías, los modelos presentados, la utilidad de la experiencia y el tiempo dedicado para su realización.

La experiencia de trabajo recibió la aprobación de una mayoría significativa. El $65 \%$ del alumnado manifestaron su satisfacción por el trabajo realizado. Afirmaron que la parte más interesante eran los modelos de presentación de los trabajos. Más del $90 \%$ del total había utilizado ese modelo de presentación, mientras que el resto de la guía, por ejemplo, en el caso de la publicidad había sido leída por algo más del $70 \%$ del alumnado. 
Los mejores resultados obtenidos se refieren al objetivo principal del trabajo, ya que en el caso de los audiovisuales el 78,8\% de los resultados válidos manifestaron que el análisis les había servido para utilizar un audiovisual (en ese curso se utilizaron exclusivamente programas de televisión de ficción) para obtener información histórica sobre el período en el que fue realizado, así como para comprender las transformaciones sociales. Los resultados en el caso del análisis de la publicidad fueron algo inferiores, aunque también positivos, con un $64 \%$ de satisfacción.

En la valoración final de la actividad, en el apartado referido a señalar los aspectos positivos de la experiencia, a los alumnos y alumnas les pareció interesante $(20,6 \%$ y $30 \%$ ), didáctica $(24,6 \%$ y $11,4 \%)$, y divertida $(19,4 \%$ y $12 \%)$. Un $10,5 \%$ en el caso de la televisión y un $17,1 \%$ en el de la publicidad, manifestaron que a partir de la experiencia han empezado a abordar este tipo de medios de comunicación con una mirada diferente.

En definitiva, estos resultados indicaron que se había mejorado la actividad docente y que los estudiantes habían incorporado una mirada diferente sobre alguno de los más populares medios de comunicación, utilizando un método de análisis riguroso.

En cuanto a la dedicación necesaria, los resultados fueron los esperados. La mayoría de los estudiantes había utilizado entre 4 y 8 horas de trabajo personal y entre 3 y 6 de trabajo en grupo. Un porcentaje importante de estudiantes trabajaron por encima de la media, dedicando más tiempo de lo que el trabajo requería. Esto indica tanto un alto nivel de interés y compromiso con el trabajo, traducido en una mejora significativa de los resultados, como en otros casos las dificultades del trabajo en grupo experimentadas por algunos alumnos.

También hay que señalar los aspectos negativos detectados. Una parte de los estudiantes declaró que no había leído ninguna de las dos guías. Esta circunstancia puede ser explicada por diversos motivos, el más importante en relación a la dinámica de trabajo en grupo, ya que no todos los miembros de un grupo consideran necesaria la lectura de las guías, optando por informarse a través del resto de compañeros y compañeras de su grupo de trabajo. Para solucionar esta situación se planteó la reducción de las dimensiones de las guías, para no inhibir a los menos dispuestos. Igualmente, se consideró la necesidad de aumentar el número de tutorías de seguimiento del desarrollo de los trabajos. Estas y otras mejoras se han ido incorporando en los cursos posteriores.

\section{Conclusiones}

Este artículo ha presentado un método didáctico de análisis de la publicidad y de la ficción que se ha experimentado en las asignaturas de Historia de la Comunicación Social en las carreras de Periodismo, Comunicación Audiovisual y Publicidad y Relaciones Públicas de la UAB. El método fue evaluado por los estudiantes, como 
muestra el apartado anterior. Este procedimiento permitió mejorar la herramienta metodológica y las guías didácticas que se ofrecen a los estudiantes para llevar a cabo el trabajo encomendado.

La gran calidad de los trabajos finales entregados por los estudiantes, confirman la validez de la metodología y de la propuesta de innovación docente desarrollada, revelando que la cultura popular es también una fuente histórica imprescindible. La descripción de los diferentes personajes, de sus actuaciones y de los escenarios en que estas se dan, de forma sistemática, ordenados siguiendo las etapas de la vida y diferenciándolos según otras características sociales, permite apreciar la diversidad social, y las relaciones que se establecen entre las historias personales y la historia colectiva.

Así, los resultados de esta experiencia docente nos llevan a poder validar su utilización como material didáctico en el campo de la enseñanza de la Historia y de las Ciencias de la Comunicación, específicamente en el ámbito de la alfabetización digital y del análisis crítico de contenidos mediáticos. Además, también puede resultar muy útil en otras disciplinas de las Ciencias Sociales, ya que muestran como los productos mediáticos y de la cultura popular son una muy importante fuente histórica, Estos productos mediáticos ofrecen otras lecturas sociales y pueden ser utilizados con la finalidad de propiciar ciudadanía, democracia y participación ya que desvelan, más allá de aspectos formales o estéticos, explicaciones más amplias sobre las que debatir. Así, muestran la diversidad de circunstancias que se dan en las vidas de las personas en relación a los contextos comunes, mostrando los cambios y transformaciones sociales como resultado tanto del esfuerzo colectivo como del individual y de las actuaciones institucionales

Comparar los resultados del análisis de los productos audiovisuales con otras fuentes históricas permite refrendar los resultados, además de incorporar la perspectiva y los intereses de otros seres humanos anónimos y próximos. Así, los estudiantes pueden ver cómo es de vital importancia que en la construcción del conocimiento social se tengan en cuenta también los saberes y la memoria de las personas individuales que han construido sus propios conocimientos a partir de la experiencia directa y de la socialización de los medios de comunicación. Este procedimiento, junto con la forma de presentación y exposición de los trabajos configura un método cooperativo de construcción de conocimiento en red, incorporando al análisis tanto saberes expertos como vivenciales.

Esta metodología de análisis puede también ser aplicada en otros niveles educativos y para analizar otros productos de la cultura popular (literatura, cómics, música, etc.). El resultado primordial es ofrecer unas herramientas para tener en cuenta otras fuentes que no son las institucionales o las consideradas "serias" por la historiografía, pero que son consumidas por una gran cantidad de personas y que definen y socializan su vida diaria y colectiva. En esta línea es necesario que historiadores e investigadores de la comunicación unan sus esfuerzos y preparen propuestas que permitan enriquecer el análisis de la vida social en el pasado y el presente. 


\section{Bibliografía}

AMBRÓS, A.; BREU, R. (2007). Cine y Educación. El cine en el aula de primaria y secundaria. Barcelona: Grao.

ARREGUI, J. A. (2009). Por el cambio. 30 años de propaganda política en España. Sevilla: Comunicación Social.

BUCKINGHAM, D. (2003). Media Education: Literacy, Learning and Contemporary Culture. Cambridge: Polity Press.

COLÓN, E. (2001). Publicidad y hegemonía. Matrices discursivas. Buenos Aires: Norma.

FOWLES, J. (1996). Advertising and Popular Culture. Thousand Oaks, CA: Sage.

FRANCISCO, A. (2010). "Construyendo ciudadanía participativa en la sociedad de la información”. En Píxel-Bit. Revista de Medios y Educación, no 37. p. 135-146. http://www.sav.us.es/pixelbit/pixelbit/articulos/n37/11.pdf. [06-10-2011].

GÓMEZ, F. J. (2007). "Narrativa cinematográfica y enseñanza del cine". En: Comunicar, $\mathrm{n}^{\circ}$ 29. p. 75-80. http://www.revistacomunicar.com/index.php?contenido=detalles\&numero=29\&articulo=29-2007-13. [12-11-2011].

(2010). El análisis de textos audiovisuales. Significación y sentido. Santander: Shangri-La Ediciones. Disponible en: http://www.shangrilaediciones.com/Materiales3-El-Analisis-Textos-Audiovisuales.pdf. [06-10-2011]

HERNÁNDEZ, S. (ed.). 2012. La Guerra Civil televisada. La representación de la contienda en la ficción y el documental españoles. Salamanca: Comunicación Social.

KELLNER, D.; SHARE, J. (2007). "Critical Media Literacy, Democracy, and the Reconstruction of Education", En MACEDO, D.; STEINBERG, S. R. (Eds.). Media literacy: A reader. New York: Peter Lang Publishing. p. 3-23.

IBÁÑEZ, J. C.; ANANIA, F. (2010). Memoria histórica e identidad en cine y televisión. Zamora: Comunicación Social.

MARZAL, J. (2007). "El análisis filmico en la era de las multipantallas". En: Comunicar, $\mathrm{n}^{\circ}$ 29. p. 63-68. http://www.revistacomunicar.com/index.php? contenido=detalles\&numero=29\&articulo=29-2007-11. [10-10-2011].

MEDRANO, M. C. (2008). "Los hábitos y la dieta televisiva en distintas edades: implicaciones educativas". En: Píxel-Bit. Revista de Medios y Educación, no 31 . p. 59-70. http://www.sav.us.es/pixelbit/pixelbit/articulos/n31/n31 art/art315.htm. [10-10-2011].

MOLINA, P. (2007). Encontrarse en la Historia. Fuentes, protagonistas y espacios: metodologías para una historia de la comunicación no-androcéntrica. Barcelona: Servei de Publicacions de la UAB. http://www.tesisenxarxa.net/TDX-1213107105836. [12-10-2011]

MONTERO, M. (Coord.) (2010). Historia de la publicidad y de las relaciones públicas en España. Zamora: Comunicación Social.

MORENO, A.; ROVETTO, F.; BUITRAGO, A. (2007). ¿De quién hablan las noticias? Guía para humanizar la información. Barcelona: Icaria.

MORENO, A. (1986): El Arquetipo Viril protagonista de la historia. Ejercicios de lectura no-androcéntrica. Barcelona: LaSal. 
(1998). La mirada informativa. Barcelona: Bosch.

(1999). Cap a una història de la comunicació des de Catalunya, ex-céntrica, plural i interactiva. Barcelona: Servei de Publicacions de la UAB.

NAVARRETE, L. (2009). La historia contemporánea de España a través del cine español. Madrid: Síntesis.

NÚÑEZ, T. (2010). "Mujeres directoras de cine: un reto, una esperanza". En: PixelBit. Revista de Medios y Educación, no 37. p. 121-133. http://www.sav.us.es/pixelbit/pixelbit/articulos/n37/10.pdf. [10-10-2011].

PALAO, J. A. (2007). "Alfasecuenciación: la enseñanza del cine en la era del audiovisual". En: Comunicar, n ${ }^{\circ} 29$. p. 87-93. http://www.revistacomunicar.com/index. php? contenido $=$ detalles\&numero $=29 \&$ articulo $=29-2007-15 .[11-11-2011]$.

PÉREZ, J. M. (2011). "El aprendizaje de la lectura comprensiva y crítica". En: Aula de Innovación Educativa, $\mathrm{n}^{\circ} 200$; p. 55-59.

ROVETTO, F. (2010). La representación del trabajo de las mujeres en la prensa: Análisis comparativo y cualitativo de la información de actualidad. Barcelona: Servei de Publicacions de la UAB. http://www.tdx.cat/TDX-1222110-182743. [12-11-2011]

SANCHEZ-BIOSCA, V. (2006). Cine de historia, cine de memoria. La representación y sus límites. Madrid: Cátedra.

SIMELIO, N. (2007). Prensa de información general durante la transición politica (1974-1984): pervivencias y cambios en la representación de las relaciones sociales. Barcelona: Servei de Publicacions de la UAB. http://www.tdx.cat/ TDX-0327107-160534. [12-11-2011]

SOPEÑA, A. (1994). "La publicitat com a font documental per a la història comtemporània. El Hogar y la Moda (1913-1931)". En: Gazeta, no 1. p. 359-369. http:// www.raco.cat/index.php/Gazeta/article/view/246389/330150. [09-10-2011].

VALERO, T. (2010). Historia de España contemporánea vista por el cine. Barcelona: Publicacions i Edicions de la Universitat de Barcelona.

\section{Los autores}

Pedro Molina Rodríguez-Navas es profesor asociado del Departamento de Periodismo y de Ciencias de la Comunicación de la UAB. Es también investigador del "Laboratorio de Periodismo y Comunicación para la Ciudadanía Plural" de la UAB. Sus líneas de investigación y publicaciones principales abordan el papel de la comunicación en democracia, específicamente en relación a participación ciudadana, comunicación pública, educación, vínculos entre memorias personales y memoria colectiva y sobre los medios y redes de comunicación como agentes constructores de memoria social.

Núria Simelio Solà es Profesora Agregada del Departamento de Periodismo y de Ciencias de la Comunicación de la UAB. Es miembro de los grupos de investigación "Observatorio Internacional de Televisión (OITVE)" y del "Laboratorio de Comunicación para la Ciudadanía Plural de la UAB”. Sus líneas de investigación y publica- 
ciones más destacadas se centran en el análisis de las representaciones sociales, étnicas y de género en los medios de comunicación. Complementariamente, una de sus líneas de investigación principal parte del estudio de las nuevas formas de producción de contenidos y de las prácticas sociales en Internet y en las redes sociales digitales.

Jordi Ibarz Gelabert es profesor lector del Departamento de Historia Contemporánea de la Universidad de Barcelona. Es miembro de "Trans-net", grupo de innovación docente de la UB. Sus líneas de investigación sobre innovación docente se centran en el desarrollo del pensamiento analítico y en la utilización de fuentes históricas con finalidades docentes. Ha trabajado en la articulación entre las memorias personales y familiares y la construcción del discurso histórico. 